\title{
Phase analysis of quantum oscillations in graphite
}

\author{
Igor A. Luk'yanchuk ${ }^{1,2}$ and Yakov Kopelevich ${ }^{3}$ \\ ${ }^{1}$ University of Picardie Jules Verne, Laboratory of Condensed Matter Physics, Amiens, 80039, France \\ ${ }^{2}$ L. D. Landau Institute for Theoretical Physics, Moscow, Russia \\ 3 Instituto de Fsica "Gleb Wataghin", Universidade Estadual de Campinas, \\ Unicamp 13083-970, Campinas, Sao Paulo, Brazil
}

(Dated: November 18, 2021)

\begin{abstract}
The quantum de Haas van Alphen (dHvA) and Shubnikov de Haas (SdH) oscillations measured in graphite were decomposed by pass-band filtering onto contributions from three different groups of carriers. Generalizing the theory of dHvA oscillations for 2D carriers with arbitrary spectrum and by detecting the oscillation frequencies using a method of two-dimensional phase-frequency analysis which we developed, we identified these carriers as (i) minority holes having a 2D parabolic massive spectrum $p_{\perp}^{2} / 2 m_{\perp}$, (ii) massive majority electrons with a $3 \mathrm{D}$ spectrum and (iii) majority holes with a $2 \mathrm{D}$ Dirac-like spectrum $\pm v p_{\perp}$ which seems to be responsible for the unusual strongly-correlated electronic phenomena in graphite.
\end{abstract}

PACS numbers: 81.05.Uw, 71.20.-b

Studies of electronic properties of graphite have considerably intensified during the past decade because of the discovery of novel carbon-based materials such as fullerenes and nanotubes constructed from wrapped graphite sheets [1]. The quasi 2D conductivity of graphite occurs mostly inside the carbon layers due to hexagonal networks of overlapped $\pi$-bonds. In this single-layer approximation the Fermi surface (FS) is reduced to two points at the opposite corners of the $2 \mathrm{D}$ hexagonal Brillouin zone where the valence and conducting bands touch each other leading to the Dirac cone spectrum $E(p)= \pm v p_{\perp}$, and the charge carriers are described by the massless $(2+1)$ dimensional Dirac fermions 2, 3, 4]. This point-like spectrum singularity and strong Coulomb coupling between fermions are assumed to be responsible for unusual electronic features in graphite such as, e. g., experimentally observed magnetic-fielddriven metal-insulator transition [5, 6].

However, to the best of our knowledge no unambiguous experimental evidence for Dirac fermions in graphite has been yet reported. In real graphite samples the interlayer hopping leads to $p_{z}$-spectrum dispersion with opening of cigar-like FS pockets elongated along the corner edge H-K-H of the 3D hexaedronal Brillouin zone. The discussed in detail in Refs. 77, 8, 9] 3D FS has a complicated multi-sheet structure and provides for the different groups of carriers. Band calculations show that in addition to the two principle majority groups of electrons (e) and holes (h) which are located close to points $\mathrm{K}$ and $\mathrm{H}$ of the Brillouin zone, several minority $(\mathrm{m})$ lowconcentration groups carriers are possible. The nature and location of the minority pockets are very sensitive to the parameters of the band structure calculations and to the crystalline disorder.

One can expect that the model of strongly interacting 2D Dirac fermions is applicable to the real quasi 2D FS in graphite since the Dirac singularity is the topologi- cal property of the electronic spectra [10] that should be stable towards the weak 3D inter-layer coupling.

In this Letter we make a comparative phase analysis of quantum de Haas van Alphen (dHvA) oscillations of the magnetization $M(H)$ and of Shubnikov de Haas ( $\mathrm{SdH}$ ) oscillations of the resistance $R(H)$ which provides direct evidence that the group of carriers, associated with majority holes (h) has the Dirac singularity in the spectrum. Other groups: (e and $\mathrm{m}$ ) have the massive spectrum $E(p)=p_{\perp}^{2} / 2 m_{\perp}$.

Quantum dHvA and SdH oscillations are the appropriate tools to study FS properties and to distinguish between different types of fermion carriers. Early measurements of dHvA and SdH oscillations [7, 8, 9, 11] in agreement with band structure calculations demonstrated that two majority (e and h) and at least one minority $(m)$ group of carriers exist in graphite.

To discriminate between normal, i. e. described by the massive spectrum, and Dirac fermions, we explored the rarely measured phase of quantum oscillations. Generally, phase detection encounters difficulties related to interference of the contributions from different groups of carrier and to its sensitivity to errors in frequency determination. We overcame the problem using a speciallydeveloped two-dimensional phase-frequency analysis of its Fourier image. We distinguish also the different carrier groups by applying selective pass-band filtering of the oscillating signal.

The magnetoresistance $R(H)$ and magnetization $M(H)$ data were obtained on well-characterized highly oriented pyrolytic graphite (HOPG) sample from the Union Carbide Co (HOPG-UC) as described in Ref. [5]. Briefly, low-frequency $(1 \mathrm{~Hz})$ and dc standard fourprobe magnetoresistance measurements were performed in magnetic field $0-90 \mathrm{kOe}$ applied parallel to the sample hexagonal c-axis $(H \| c)$, and at the lowest available temperature $T=2 \mathrm{~K}$ using Quantum Design PPMS-9T 

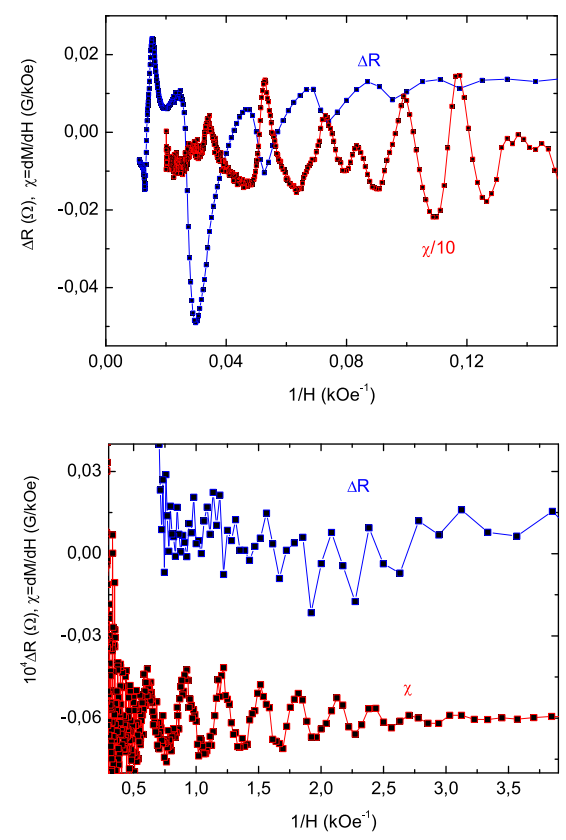

FIG. 1: dHvA and SdH oscillations in graphite. Upper panel shows the region of fields $7 k O e<H<50 k O e$, characteristic for majority oscillations whereas the region of fields in low panel $0.25 k O e<H<2.5 k O e$ corresponds to minority oscillations

and Janis-9T magnet He-cryostats. Magnetization measurements $M(H)$ were carried out with $H \| c$ by means of the SQUID magnetometer MPMS5 (Quantum Design).

Fig [1] shows the measured magnetic susceptibility $\chi=$ $d M / d H$ and the oscillating part of resistance $\Delta R$ (after substraction of the large polynomial background $R_{0}(H)$ ) as a function of the inverse magnetic field $H^{-1}$ in the high- and low-field regions. In agreement with previous experiments [7, 8, 9, 11], $\chi\left(H^{-1}\right)$ is a superposition of at least three oscillating contributions. This can be seen in Fig. 2 where both principal peaks $m_{1}, e_{1}$ and $h_{1}$ and their second harmonic counterparts $m_{2} e_{2}$ and $h_{2}$ in spectral intensity of Fourier transformed susceptibility $|\chi(\nu)|$ are plotted. At the same time, only the $m$ and $e$ peaks are seen in the spectral intensity of resistance $|R(\nu)|$. In other words, the $\mathrm{SdH} h$-oscillations are strongly damped. The corresponding oscillation frequencies $\nu_{i}$ and their assignment to the different groups of carries which we justify below, are given in Table I. Note that unlike the widely accepted result $\nu_{h}<\nu_{e}$ [1], the hole frequency $\nu_{h}$ in our sample is higher then the electron frequency $\nu_{e}$.

We decomposed the measured signals $\chi\left(H^{-1}\right)$ and $R\left(H^{-1}\right)$ onto individual $m$-, $e$ - and $h$-oscillations, applying the frequency filtering with selective pass-bands about the 1st and 2nd harmonics of the corresponding resonant frequencies $\nu_{i}$. As shown in Fig. 3 the results demonstrate the generic behavior for quantum oscillations: an initial growth of the high-field amplitude (low

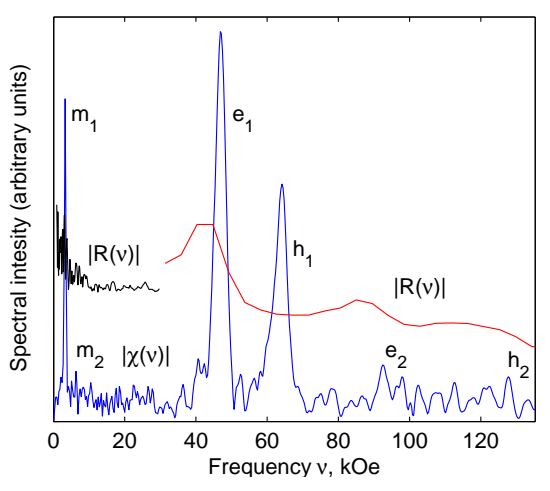

FIG. 2: Spectral intensity of dHvA oscillations of susceptibility $|\chi(\nu)|$ and of SdH oscillations of magnetoresistance $|R(\nu)|$. Peaks $m_{1,2}, e_{1,2}, h_{1,2}$ correspond to the 1st and 2nd harmonics of oscillations from minority electrons, majority electrons and majority holes. The low and high frequency plots of $|R(\nu)|$ are obtained from different sets of experimental data.

TABLE I: Frequencies $\nu_{i}$ and phases $\phi_{i}$, phase factors $\mu_{i}, \gamma_{i}$, $\delta_{i}$ and assignment of quantum oscillations in graphite.

\begin{tabular}{lccrccc}
\hline \hline & $\nu_{i}(\mathrm{kOe})$ & $\phi_{i}$ & $\mu_{i}$ & $\gamma_{i}$ & $\delta_{i}$ & Assignment \\
\hline minority m & 3.28 & 0 & -1 & $1 / 2$ & 0 & normal h, 2D \\
majority e & 46.8 & $0.75 \pi$ & 1 & $1 / 2$ & $-1 / 8$ & normal e, 3D \\
majority h & 64.1 & $\pi$ & -1 & 0 & 0 & Dirac h, 2D \\
\hline \hline
\end{tabular}

Landau levels) followed by a low-field Dingle attenuation $\sim e^{-A_{i} / H}$. The low-intensity $\mathrm{SdH}$ h-oscillation are recovered from the noisy background of $R(\nu)$ around $\nu_{h}$. The sign of $\Delta R$ in Fig. [3] is reversed in order to recover the behavior of oscillating part of the conductivity $\Delta \sigma=\Delta\left(\rho^{-1}\right) \approx-\Delta \rho / \rho_{0}^{2} \sim-\Delta R$.

To proceed with the phase detection, we analyze how the nature of the carriers influences the phase of the quantum oscillations, by considering the quasi 2D spectrum appropriate for graphite:

$$
\varepsilon(\mathbf{p})=\varepsilon_{\perp}\left(p_{\perp}\right)-2 t \cos p_{z} d \pm \mu_{B} H,
$$

where the perpendicular dispersion $\varepsilon_{\perp}\left(p_{\perp}\right)$ can be either of the massive (parabolic) or of the Dirac (linear) type (see Table II); $\pm \mu_{B} H$ is the Zeeman splitting $\left(\mu_{B}=\right.$ $e \hbar / 2 m c)$.

The original theory of dHvA oscillations of Lifshitz and Kosevich [12] was developed for 3D metals with an arbitrary dispersion $\varepsilon(p)$, applied to the spectrum (1) when the energy spacing $\hbar \omega_{c}$ between Landau levels at the FS is smaller then the characteristic dispersion $t$ along $z$. The other limit of almost 2D electrons was studied quite recently and the general expression that incorporates both $\hbar \omega_{c} \ll t$ and $\hbar \omega_{c} \gg t$ limits was derived in [13] for the case of parabolic dispersion of $\varepsilon_{\perp}\left(p_{\perp}\right)$. It is straightforward to generalize the calculations of 13] for the case of arbitrary dispersion, using as in [12] the elec- 

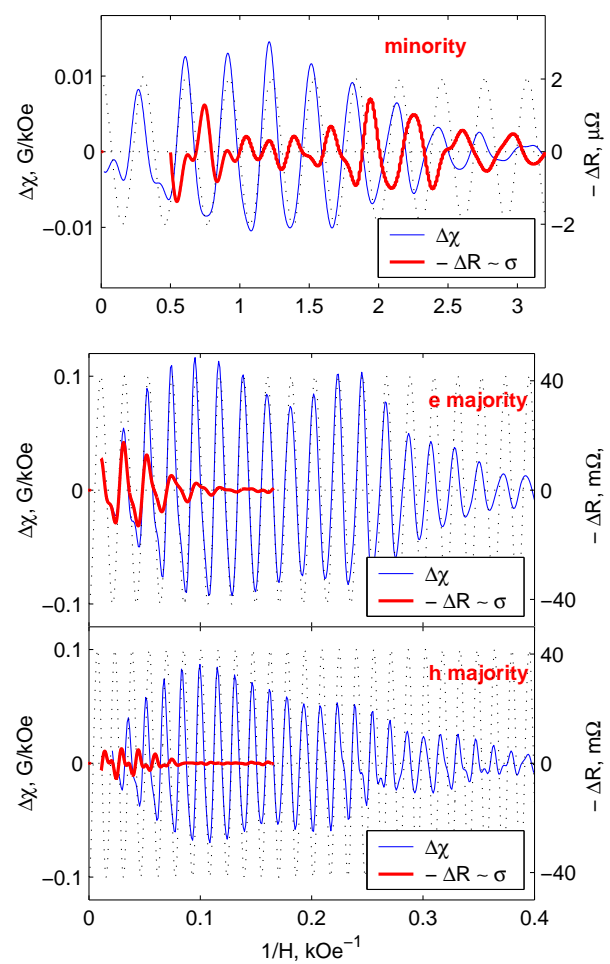

FIG. 3: Quantum oscillations of susceptibility $\Delta \chi\left(H^{-1}\right)$ and of resistance $\Delta R\left(H^{-1}\right)$ for different groups of carriers, obtained after two-harmonic band-pass filtering of experimental data. Dot-lines show the one-harmonic phase fit using the phase factors specified in Table \The sign of $\Delta R$ is inverted to recover the oscillating part of the conductivity $\Delta \sigma$.

tron orbit area $S\left(\varepsilon, p_{z}\right)=\pi p_{\perp}^{2}\left(\varepsilon, p_{z}\right)$ instead of $\varepsilon_{\perp}\left(p_{\perp}\right)$ and the Bohr-Sommerfeld semiclassical quantization:

$$
S\left(\varepsilon, p_{z}\right)=(n+\gamma) 2 \pi \hbar \frac{e H}{c},
$$

where the factor $0 \leq \gamma<1$ is related to the topology of the FS: $\gamma=1 / 2$ for parabolic dispersion and $\gamma=0$ in the Dirac case [10].

Reproducing the calculations of 13 in terms of $S\left(\varepsilon, p_{z}\right)$ we get for the oscillating part of the magnetization:

$$
\begin{aligned}
& \Delta M=-\frac{4}{(2 \pi)^{2}} \frac{1}{\hbar d} \frac{e}{\hbar c} \frac{S}{d S / d \varepsilon} \sum_{l=1}^{\infty} \frac{1}{l} \frac{\lambda l}{\sinh \lambda l} e^{-\frac{\Gamma}{\hbar \omega_{c}} 2 \pi l} \\
& \times J_{0}\left(2 \pi l \frac{2 t}{\hbar \omega_{c}}\right) \sin \left(2 \pi l\left[\frac{c}{e \hbar} \frac{S(\varepsilon)}{2 \pi H}-\gamma\right]\right) \cos \left(2 \pi l \frac{\mu_{B} H}{\hbar \omega_{c}}\right),
\end{aligned}
$$

where $\Gamma$ is the impurity width of the Landau level and parameters

$S(\varepsilon)=S(\varepsilon, \pi / 2 d), \quad \lambda=\frac{\pi c T}{e \hbar H} \frac{d S}{d \varepsilon} \ll 1, \quad \omega_{c}=\frac{e H}{c} \frac{2 \pi}{d S / d \varepsilon}$

are given in Table 1 for the normal and Dirac fermions.

Calculated with respect to the band origin (at $p_{z}=$ $0)$ the chemical potential $\epsilon$ equilibrates the oscillating
TABLE II: Spectra, Landau quantization, areas of the quasiclassical electronic orbits and parameters of the dHvA oscillations for the massive (Normal) and masseless (Dirac) fermions

\begin{tabular}{lll}
\hline \hline & Normal & Dirac \\
\hline$\varepsilon(\mathbf{p})$ & $p_{\perp}^{2} / 2 m_{\perp}$ & $\pm v\left|p_{\perp}\right|$ \\
$\varepsilon_{\perp}(n)$ & $\left(e \hbar / m_{\perp} c\right) H\left(n+\frac{1}{2}\right)$ & $\pm\left(2 v^{2} e \hbar / c\right)^{1 / 2}(H n)^{1 / 2}$ \\
$S(\varepsilon)$ & $2 \pi m_{\perp} \varepsilon$ & $\pi \varepsilon^{2} / v^{2}$ \\
$\omega_{c}$ & $e H / c m_{\perp}$ & $e v^{2} H / c \varepsilon$ \\
$\lambda$ & $2 \pi^{2} c m_{\perp} T / e \hbar H$ & $2 \pi^{2} c T \varepsilon / \hbar e v^{2} H$ \\
\hline \hline
\end{tabular}

Fermi-levels of different groups of carriers and therefore acquires the field dependence that was shown [14] to be important for the very clean 2 D systems with $\omega_{c} \gg \Gamma$ and in the ultra-quantum limit when only low Landau levels $(n \sim 1)$ are occupied. In the opposite case, we neglect this dependence and assume that $\epsilon=\epsilon_{F}$. Note, however, that beats in the majority oscillations (Fig. 3) can be attributed to the conserving $\epsilon$ "cross-talk" between e and $\mathrm{h}$ carriers [15]. We neglect also the last oscillating spin factor in (3) since the Zeeman splitting $\mu_{B} H$ in graphite is much smaller than the distance between Landau levels $\hbar \omega_{c}$ 11. This tiny splitting feature is observed only in the high-field e-oscillations in Fig 3

Equation (3) includes both the 3D Lifshitz-Kosevich limit [2] when $\zeta=2 \pi l \frac{2 t}{\hbar \omega_{c}} \gg 1, J_{0}(\zeta) \approx$ $(2 / \pi \zeta)^{1 / 2} \cos (\zeta-\pi / 4)$ and pure $2 \mathrm{D}$ limit when $\zeta \ll 1$, $J_{0}(\zeta) \approx 1$. In the case of $2 \mathrm{D}$ Dirac fermions it reduces to the result obtained in Ref. [16], whereas in the case of massive fermions the result of [13] is recovered. Analyzing only the oscillating part of $\Delta M$, we find that the lst harmonic of the magnetic susceptibility $\Delta \chi=d(\Delta M) / d H$ oscillates as:

$$
\Delta \chi_{l} \sim \mu \cos \left(2 \pi\left[\frac{\nu}{H} l-\gamma l+\delta\right]\right)
$$

where the factor $\mu=\operatorname{sign}(\epsilon)$ is equal to +1 for the electrons and to -1 for the holes. The topological index $\gamma$ (see Eq. (2)) is equal to $1 / 2$ for massive fermions and is 0 for Dirac fermions. The factor $\delta$ reflects the curvature of the FS in the z-direction and changes from 0 for a quasi-2D cylindrical FS when $\hbar \omega_{c} \ll t$ to $\pm \pi / 8$ for a corrugated 3D FS when $\hbar \omega_{c} \gg t$ ( \pm corresponds to the contribution from minimal/maximal cross section).

In order to determine factors $\mu_{i}, \gamma_{i}$ and $\delta_{i}$ for each group of presented in Fig. 3 oscillations we extract the phase of their 1st harmonics $\varphi_{i}$, analyzing Fouriertransformed susceptibility $\chi(\nu)$ in the vicinity of oscillation frequencies $\nu_{i}$. Because the phase information can not be extracted from the spectral intensity plot $|\chi(\nu)|$ (Fig. 22), we developed the method of the 2D phase-frequency analysis that is free from the above mentioned phase/frequency uncertainty. For its illustration we assume that close to the resonance frequency $\nu_{0}$ the 


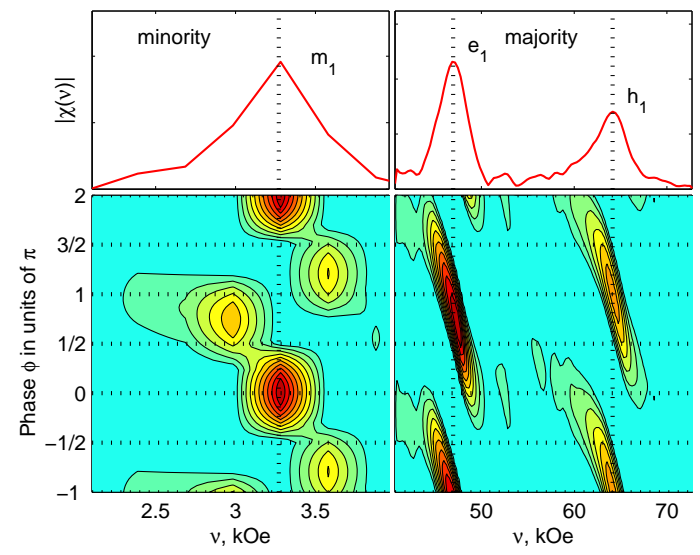

FIG. 4: Contour plot of the positive part of the phase-shift function $K(\phi, \nu)=\operatorname{Re}^{i \varphi} \chi(\nu)$ for minority and majority carries. Position of maxima of $K(\phi, \nu)$ determines the oscillation frequencies $\nu_{i}$ and phases $\phi_{i}$ for different groups of carriers. Upper panel presents the corresponding spectral intensity $|\chi(\nu)|$.

Fourier-transformed susceptibility has the Gaussian-like profile $\chi(\nu) \sim e^{i \varphi_{0}} e^{-\beta\left(\nu-\nu_{0}\right)^{2}}$. Constructing now the phase-shift function $K(\varphi, \nu)=\operatorname{Re} e^{-i \varphi} \chi(\nu)$ that in our model case is equal to $e^{-\beta\left(\nu-\nu_{0}\right)^{2}} \cos \left(\phi-\phi_{0}\right)$ we can detect both the frequency $\nu_{0}$ and the phase $\varphi_{0}$ simultaneously as the position of the maximum of $K(\varphi, \nu)$ in the plane $\phi-\nu$.

The phase-shift function for the dHvA oscillations in graphite is shown in Fig. 4. Determination of maxima of $K(\varphi, \nu)$ gives the collected in Table $\llbracket$ oscillation frequencies $\nu_{i}$ and their phases $\varphi_{i}$ more precisely than the previous determination: $\left(\phi_{e}=\phi_{h}=0.75 \pi\right.$ in [8] and $\phi_{h}=0.76 \pi, \phi_{e}=0.64 \pi$ in $\left.[9]\right)$.

By analyzing the oscillations of $\Delta \sigma$ and $\Delta \chi$ (Fig. 3), and taking into account the relation $\Delta \sigma \sim \mu|m| H^{2} \Delta \chi$ [17, we conclude that the in- and out-of-phase behavior corresponds to electrons and holes with $\mu=+1$ and $\mu=-1$, respectively. For h-carriers this analysis was independently supported by a comparison between the longitudinal resistance oscillations $\Delta R$ and of the Hall resistance $\Delta R_{H}$ (not shown): for holes, minima in $\Delta R$ should correspond to minima in $\Delta R_{H}$ [1], and this is what we observed.

Knowledge of $\phi_{i}$ and $\mu_{i}$ allows the unambiguous determination of the factors $\gamma_{i}\left(\gamma_{i}=1 / 2\right.$ or 0$)$ and $\delta_{i}$ $\left(\left|\delta_{i}\right|<1 / 8\right)$ that are interrelated as:

$$
\phi_{i}=\pi\left(\operatorname{sign} \mu_{i}-2 \gamma_{i} l+2 \delta_{i}\right)
$$

The analysis of the 1 th harmonic parameters $\mu_{i}, \gamma_{i}$ and $\delta_{i}$, given in Table \ for each group of carriers, led us to the following conclusions. (i) The minority carriers are holes with a $2 \mathrm{D}$ massive parabolic spectrum.

(ii) The majority electrons have the parabolic spectrum with $3 \mathrm{D} p_{z}$-dispersion.

(iii) The majority holes are 2D Dirac fermions.

The identification of Dirac fermions which can be responsible for unusual strongly-correlated electronic phenomena in graphite [5, 6] is the principal result of this work.

The method proposed here for the two-dimensional phase-frequency analysis allows the efficient phase definition in any quantum oscillation phenomena, including those in low-dimensional organic conductors, in the mixed state of superconductors and in 2D quantum Hall semiconductors.

The work was supported by FAPESP and $\mathrm{CNPq}$ Brazilian scientific agencies. I.L. thanks J.-L. Dellis for discussion of computation problems.

[1] R. Saito, G. Dresselhaus, and M.S. Dresselhaus, Physical Properties of Carbon Nanotubes (Imperial College Press, London,(1998).

[2] J. González, F. Guinea, and M. A. H. Vozmediano, Phys. Rev. Lett. 77, 3589 (1996)

[3] A. A. Abrikosov, Phys. Rev. B60, 4231 (1999)

[4] D.V. Khveshchenko, Phys. Rev. Lett., 87, 206401 (2001); ibid. 87, 246802 (2001)

[5] Y. Kopelevich, J.H.S. Torres, R.R. da Silva, et al., Phys. Rev. Lett. 90, 156402 (2003).

[6] Y. Kopelevich, et al., Advances in Solid State Physics 43, 207 (2003)

[7] N. B. Brandt, S.M. Chudinov and Ya. G. Ponomarev, Semimetals I. Graphite and its Compounds, Amsterdam, (1988); B. T. Kelly, Physics of Graphite, Appl. Sci. Publ., London and New Jersey, 1981 and refs. therein.

[8] D. E. Soule, J. W. McClure, and L. B. Smith, Phys. Rev. 134, A453 (1964)

[9] S.J. Williamson, S. Foner, and M.S. Dresselhaus, Phys. Rev. 140, A1429 (1965)

[10] G. P. Mikitik and Yu.V. Sharlai, Phys. Rev. Lett. 82, 2147 (1999)

[11] J. A. Woollam Phys. Rev. B4, 3393 (1971)

[12] I. M. Lifshitz and A. M. Kosevich, Zh. Éksp. Teor. Fiz. 29, 730 (1955) [Sov. Phys. JETP 2, 636 (1956)].

[13] T. Champel and V. P. Mineev, Philos. Mag. B81, 55 (2001)

[14] T. Champel, Phys. Rev. B 64, 054407 (2001)

[15] A. S. Alexandrov and A. M. Bratkovsky Phys. Rev. Lett. 76, 1308 (1996)

[16] S.G. Sharapov, V.P. Gusynin and H. Beck, Phys. Rev. B69, 075104 (2004).

[17] E. M. Lifshitz and L. P. Pitaevskii, Physical Kinetics (Butterworth-Heinemann, Oxford, 1995) 